\title{
Solubility Estimation for Drugs Treated with the Simple Suspension Method Using Available Dissolution Test Profiles
}

\author{
Wataru Sakurada, Tetsuya Shimoyama, Kunihiko Itoh and Michiya Kobayashi* \\ School of Pharmaceutical Sciences, Health Sciences University of Hokkaido \\ $\left[\begin{array}{l}\text { Received March 19, } 2015 \\ \text { Accepted May 28, } 2015\end{array}\right]$
}

The simple suspension method (SSM) is a method of administering drugs via feeding or gastrostomy tubes to those who have difficulty swallowing. In the SSM, solid formulations (eg, tablets, capsules) are immersed in hot $\left(55^{\circ} \mathrm{C}\right)$ water. This promotes the disintegration and dissolution of the drugs and changes their solubility. However, pharmaceutical companies have not issued test results on the solubility and dissolution behaviors of suspended drugs prepared according to the SSM.

For this study, we chose 10 drugs, 8 listed and 2 not listed in the Japanese Orange Book, to compare the dissolution behaviors of each drug, treated vs untreated with the SSM. Dissolution was classified into three patterns: rapid (gliclazide and famotidine), moderate (propranolol, pindolol, metoprolol), and slow (furosemide, ibuprofen, glimepiride). The initial dissolution rates of the moderate-dissolution drugs increased markedly by employing the SSM. In this study, hydroxyzine capsules and phenytoin tablets, neither of which is listed in the Japanese Orange Book, were compared with different dosage/administration forms of those drugs listed in the book (ie hydroxyzine tablets and phenytoinphenobarbital combination tablets). The dissolution behavior of these drugs was not estimated from the dissolution profiles of different forms available in the book. Nevertheless, it seemed that the available data on the dissolution of drugs will be useful when pharmacists estimate the dissolution behavior of drugs administered using the SSM.

Key words — simple suspension method, dissolution behavior, Japanese Orange Book, drug information

\section{Introduction}

In today's medical practice, oral solid formulations (tablets and capsules) are excellent in portability and ingestibility. However, such formulations cannot be administered to patients who have difficulty swallowing. For those patients, medical preparations are more likely to be administered via gastrostomy tube, as liquid nutritional supplements and pharmaceutical suspensions. Kurata et al developed the simple suspension method (SSM) to administer drugs to those who are fed by gastrostomy tube. ${ }^{1)}$ The SSM does not require specific equipment or a specific process. It involves immersing tablets or capsules in hot $\left(55^{\circ} \mathrm{C}\right)$ water for $10 \mathrm{~min}$ so that the formulations are sufficiently disintegrated and suspended to administer via a gastrostomy tube to the stomach. Today, the SSM is commonly applied at many hospitals in Japan, ${ }^{2)}$ proving that the method may contribute to efficient medical practice. ${ }^{3-5)}$

The advantages include the prevention of drug loss from tablet crush and easy referring for administered drugs. ${ }^{1)}$ Nonetheless, the SSM is inappropriate for formulations that may clog feeding tubes due to their poor dispersability and for sustained-release formulations with modified designs. The SSM employability for oral formulations was summarized in a database by Kurata et $a l{ }^{1)}$ which serves as a guideline for clinical medi- 
cal staff.

The employability of the SSM and the stability of SSM-administered drugs have been discussed in many studies. Yano et $a l^{6}$ compared proprietary and generic pravastatin products, while Miyamoto $e t \mathrm{al}^{7)}$ compared amantadine and 5 other proprietary and generic products, to point out that treatment of the SSM resulted in a significant difference in tube permeability between some of proprietary and generic products. Yano et $a l^{8)}$ confirmed that 8 types of suspended drugs, including phenytoin and digoxin, were found to be stable. Suryani et $a l^{9)}$ demonstrated that suspended ester prodrugs (acemetacin, cefpodoxime proxetil) are stable individually, although their ester bond is cut when they are mixed with magnesium oxide.

Pharmaceutical companies are obliged to test developed formulations prior to their distribution on the market. However, the tests do not cover the administration of drug suspensions in hot $\left(55^{\circ} \mathrm{C}\right)$ water. Accordingly, there are no data accessible or reported on the dissolution behavior of suspended formulations in the SSM.

In previous studies, we examined the solubility of poorly water-soluble tablets (phenytoin and ibuprofen) in the SSM. ${ }^{10)}$ We found that the SSM promotes the immediate dissolution of phenytoin to the saturated concentration, whereas it promotes the gentle dissolution of ibuprofen. This might suggest that drugs which dissolve immediately when suspended may have enhanced solubility for the major ingredients in the drugs, and thus, the absorbability of the drug by the digestive canal may be affected. However, no details have been confirmed. This necessitates further studies on the passability of suspended drugs through gastrostomy tubes as well as on the solubility of those drugs, which will thus be used to determine whether the SSM can be used for the administra- tion of each drug.

Studies on the applicability of the SSM to commercially available individual oral formulations require a lot of time; thus they are impractical. The Japanese Orange Book lists dissolution test data for 688 pharmaceutical ingredients that are orally administered (as of January 10, 2015). However these data are the dissolution profile of the intact drug (untreated with the SSM), thus it is not possible to apply the estimation for drugs treated with the SSM directly. In this study, we focused on the data in the Japanese Orange Book and on interview forms, both of which report dissolution profiles of oral formulations, for the purpose of comparing treated vs untreated drugs with the SSM in terms of dissolution behavior.

Moreover, the data does not cover some ingredients, such as hydroxyzine pamoate and phenytoin. Hydroxyzine pamoate is only listed for tablets and not capsules, and phenytoin tablets are included as a combined drug with phenobarbital, but are not listed independently. We also discuss the validity of the data on one form of ingredient with respect to a different form.

\section{Materials \& Methods}

\section{Reagent}

Gliclazide tablets (20 mg, Glimicron ${ }^{\circledR} \mathrm{HA}$ ) and phenytoin $\left(100 \mathrm{mg}\right.$, Aleviatin $\left.^{\circledR}\right)$ tablets were purchased from the Dainippon Sumitomo Pharma Co, Ltd (Osaka, Japan). Famotidine tablets (10 mg, Gaster ${ }^{\circledR}$ ) were purchased from Astellas Pharma Inc (Tokyo, Japan). Propranolol hydrochloride tablets $\left(10 \mathrm{mg}\right.$, Inderal $\left.{ }^{\circledR}\right)$ and metoprolol tartrate tablets $\left(20 \mathrm{mg}\right.$, Seloken $\left.{ }^{\circledR}\right)$ were purchased from AstraZeneca KK (Osaka, Japan). Pindolol tablets (5 mg, Carvisken ${ }^{\circledR}$ ) were purchased from Alfresa Pharma Co (Osaka, Japan). Furosemide tablets 
(20 mg, Lasix ${ }^{\circledR}$ ) were purchased from Nichi-Iko Pharmaceutical Co, Ltd (Toyama, Japan). Ibuprofen tablets (100 mg, Brufen $\left.{ }^{\circledR}\right)$ were purchased from Kaken Pharmaceutical Co, Ltd (Tokyo, Japan). Glimepiride tablets $\left(1 \mathrm{mg}\right.$, Amary $\left.\mathrm{l}^{\oplus}\right)$ were purchased from Sanofi KK (Tokyo, Japan). Hydroxyzine pamoate capsules (25 mg, Atarax ${ }^{\circledR}-\mathrm{P}$ ) were purchased from Pfizer Japan Inc (Tokyo, Japan).

Famotidine was purchased from MP Bio Japan KK (Tokyo, Japan). $d l$-Propranolol hydrochloride, pindolol, gliclazide, glimepiride and phenytoin were purchased from Wako Pure Chemical Inc, Ltd (Osaka, Japan). Metoprolol tartrate was purchased from LKT Laboratories (St Paul, MC, USA). Furosemide and ibuprofen sodium salt were purchased from Sigma-Aldrich Co, LLC (Tokyo, Japan). Hydroxyzine pamoate was purchased from Alfa Aesar (Heysham, UK). All other reagents used were either of GR grade or HPLC grade.

\section{Information gathering by Japanese Orange Book}

The Japanese Orange Book provides online the profiles of drug dissolution behavior (http://www. jp-orangebook.gr.jp/index.html, April 24, 2015). We downloaded a PDF document to use as the data for the dissolution tests. The tests provided lead times of $5,10,15,30,45$ and $60 \mathrm{~min}$; for each of these, we visually measured the solubility of the drug.

\section{Simple suspension method (SSM)}

The simple suspension method, employed in accordance with the procedure suggested by Kurata et al, ${ }^{1)}$ was as follows: A drug was immersed in a disposable syringe $\left(50 \mathrm{~mL}\right.$, Terumo ${ }^{\circledR}$ syringe SS-50ESZ, Terumo Co, Tokyo, Japan) with $20 \mathrm{~mL}$ of purified water heated to $55^{\circ} \mathrm{C}$; the solution was left at room temperature for $10 \mathrm{~min}$ and then mixed by inverting the syringe 15 times.

\section{Dissolution test}

In accordance with the Japanese Pharmacopoeia 16th Edition (JP), we performed dissolution tests of the drugs by employing the paddle method (DT600, Erweka GmbH, Heusenstamm, Germany). Each drug was subject to comparison between treated and untreated (the control). $900 \mathrm{~mL}$ of 1st fluid ( $\mathrm{pH}$ 1.2, specified for the JP dissolution test) was used for the test, and the temperature of the fluid was kept at $37 \pm 0.5^{\circ} \mathrm{C}$. The paddle rotation speed was set as $50 \mathrm{rpm}$. The drug suspension treated with the SSM was injected into the dissolution test vessel, and the syringe was rinsed two times with 1 st fluid in the vessel. For the control, $20 \mathrm{~mL}$ of purified water heated to $55^{\circ} \mathrm{C}$ was added to the fluid and left at room temperature for $10 \mathrm{~min}$. Fluid was sampled at 5, 10, 15, 30, 45 and $60 \mathrm{~min}$. Purified water was added subsequently to fill in the sampled portion of the fluid. Sampled fluid ( $1 \mathrm{~mL}$ ) was filtered by Millex ${ }^{\circledR}$-LH (pore size $0.45 \mu \mathrm{m}$, Merck Millipore, Billerica, MA, USA), and it was diluted to 5 times with purified water to analyze the drug concentrations by HPLC. In the experiment of famotidine and gliclazide, sampled fluid was diluted using 2 nd fluid specified for the JP dissolution test ( $\mathrm{pH}$ 6.8) to prevent acid hydrolysis.

\section{HPLC analysis conditions}

Analysis was made by using the Shimadzu HPLC system (Kyoto, Japan), which consists of a LC-20AD pump, a SPD-20A UV detector, a SIL20A auto-sampler, a CTO-20A column oven, a DGU-20A $A_{3}$ degasser and a C-R8A chromatopac integrator. The columns used were Inertsil-ODS 
Table 1 HPLC conditions

\begin{tabular}{|c|c|c|c|}
\hline Drug & HPLC Column & Wavelength & Mobile phase \\
\hline gliclazide & ODS & $228 \mathrm{~nm}$ & $50 \mathrm{mM} \mathrm{KH}_{2} \mathrm{PO}_{4}(\mathrm{pH} 6.0):$ acetonitrile $=40: 60$ \\
\hline famotidine & ODS & $254 \mathrm{~nm}$ & $\begin{array}{l}10 \mathrm{mM} \text { 1-pentanesulfonate sodium salt/acetic acid }(\mathrm{pH} 3.0) \text { : } \\
\text { acetonitrile : methanol }=780: 190: 30\end{array}$ \\
\hline propranolol & ODS & $289 \mathrm{~nm}$ & $50 \mathrm{mM} \mathrm{KH}_{2} \mathrm{PO}_{4}(\mathrm{pH} 6.0):$ acetonitrile $=70: 30$ \\
\hline pindolol & ODS & $215 \mathrm{~nm}$ & $50 \mathrm{mM} \mathrm{KH}_{2} \mathrm{PO}_{4}(\mathrm{pH} 6.0):$ acetonitrile $=85: 15$ \\
\hline metoprolol & ODS & $222 \mathrm{~nm}$ & $50 \mathrm{mM} \mathrm{KH}_{2} \mathrm{PO}_{4}(\mathrm{pH} 6.0):$ acetonitrile $=80: 20$ \\
\hline furosemide & ODS & $229 \mathrm{~nm}$ & $50 \mathrm{mM} \mathrm{KH}_{2} \mathrm{PO}_{4}(\mathrm{pH} 6.0):$ acetonitrile $=80: 20$ \\
\hline ibuprofen & $\mathrm{C} 8$ & $225 \mathrm{~nm}$ & $50 \mathrm{mM} \mathrm{KH}_{2} \mathrm{PO}_{4}(\mathrm{pH} 6.0):$ acetonitrile $=70: 30$ \\
\hline glimepiride & $\mathrm{C} 8$ & $228 \mathrm{~nm}$ & $50 \mathrm{mM} \mathrm{KH}_{2} \mathrm{PO}_{4}(\mathrm{pH} 6.0):$ acetonitrile $=40: 60$ \\
\hline hydroxyzine & ODS & $231 \mathrm{~nm}$ & $50 \mathrm{mM} \mathrm{KH}_{2} \mathrm{PO}_{4}(\mathrm{pH} 6.0):$ acetonitrile $=50: 50$ \\
\hline phenytoin & ODS & $229 \mathrm{~nm}$ & $50 \mathrm{mM} \mathrm{KH}_{2} \mathrm{PO}_{4}(\mathrm{pH} \mathrm{6.0)}:$ acetonitrile $=80: 20$ \\
\hline
\end{tabular}

ODS: Inertsil ODS-4, C8: Inertsil C8-4.

(4.6 mm $\times 150 \mathrm{~mm}, 5 \mu \mathrm{m}$, GL Sciences Inc, Tokyo, Japan) and Inertsil-C8-4 (4.6 mm $\times 150 \mathrm{~mm}, 5$ $\mu \mathrm{m}, \mathrm{GL}$ Sciences Inc). The flow rate was 0.8 to $1.0 \mathrm{~mL} / \mathrm{min}$. The column temperature was kept at $55^{\circ} \mathrm{C}$. The column types, detected wavelengths and mobile phases of each drug are shown in Table 1. The conditions of HPLC analysis were slightly modified from those of JP and the Japanese Orange Book.

\section{Statistical analysis}

All data were presented as the mean value \pm standard deviation (SD). The difference in solubility of treated vs untreated drugs with the SSM was evaluated by Student's $t$-test, and significance was defined as $P<0.05$.

\section{Results}

\section{Dissolution behavior of drugs listed in Japanese Orange Book}

Table 2 shows the eye-measured solubility values of tested drugs, which are listed on the Japanese Orange Book website (http://www.jporangebook.gr.jp/index.html, April 24, 2015). According to Table 2, more than $80 \%$ of gliclazide and the famotidine dissolved within $10 \mathrm{~min}$, less than $50 \%$ of the propranolol, the pindolol and the metoprolol dissolved within $10 \mathrm{~min}$, and most of them dissolved in $60 \mathrm{~min}$. Furosemide, ibuprofen and glimepiride dissolved very slowly, with less than 50\% dissolution in $60 \mathrm{~min}$.

From the data, the drugs tested for this study

Table 2 Cumulative solubility (\%) of drugs from the dissolution test (1st fluid for dissolution test)

\begin{tabular}{|c|c|c|c|c|c|c|}
\hline Drug & $5 \mathrm{~min}$ & $10 \min$ & $15 \min$ & $30 \mathrm{~min}$ & $45 \mathrm{~min}$ & $60 \mathrm{~min}$ \\
\hline gliclazide tablet (20 mg) & 68 & 88 & 96 & 98 & $>99$ & $>99$ \\
\hline famotidine tablet (10 mg) & 37 & 81 & 89 & 96 & 98 & $>99$ \\
\hline propranolol $\mathrm{HCl}$ tablet (10 mg) & 28 & 43 & 54 & 84 & 98 & 98 \\
\hline pindolol tablet (5 mg) & 18 & 31 & 42 & 67 & 84 & 94 \\
\hline metoprolol tartrate tablet (20 mg) & 10 & 28 & 45 & 94 & 97 & $>99$ \\
\hline furosemide tablet (20 mg) & 5 & 15 & 19 & 30 & 34 & 39 \\
\hline ibuprofen tablet (100 mg) & 0 & 0 & 2 & 12 & 18 & 23 \\
\hline glimepiride tablet $(1 \mathrm{mg})$ & 0 & 0 & 0 & 1 & 2 & 3 \\
\hline
\end{tabular}


can be categorized into three groups by dissolution pattern: those that dissolved rapidly (the rapid-dissolution group), those that dissolved moderately and most of the formulations dissolved in the end (the moderate-dissolution group), and those that dissolved slowly (the slowdissolution group).

\section{Change in dissolution behavior of SSM- treated drugs}

Next, we analyzed the dissolution behaviors of treated vs untreated drugs with the SSM for the rapid-dissolution group, the moderate-dissolution group and the slow-dissolution group. In the rapid-dissolution group, treated gliclazide was found to have a slightly greater solubility than untreated gliclazide (Fig 1A). Treated famotidine was found to have double the solubility of untreated famotidine at $5 \mathrm{~min}$, the behavior became similar between the two in $10 \mathrm{~min}$ (Fig 1B).

In the moderate-dissolution group, treated propranolol dissolved rapidly for the first $15 \mathrm{~min}$, which showed a significant difference from untreated propranolol (Fig 2A). Treated pindolol also dissolved rapidly. A significant difference was recognized between treated and untreated pindolol in the first $5 \mathrm{~min}$, and that difference re- mained the same at 45 min (Fig 2B). In addition, metoprolol showed a great difference in solubility within $15 \mathrm{~min}$, although most of the drug dissolved in 30 min whether or not it was treated with the SSM (Fig 2C).

In the slow-dissolution group, less than $20 \%$ of treated furosemide dissolved within $5 \mathrm{~min}$, which was still more than double that for untreated furosemide (Fig 3A). Whether or not it was treated with the SSM, furosemide continued to dissolve at a constant rate, and the difference in solubility between treated and untreated drugs remained approximately $10 \%$. The solubility of treated ibuprofen was approximately 3 percentage points greater than untreated ibuprofen in $10 \mathrm{~min}$, and the difference remained the same subsequently (Fig 3B). The solubility of glimepiride was approximately 2 percentage points different for treated vs untreated at $5 \mathrm{~min}$, the difference narrowing eventually to nil at $30 \mathrm{~min}$ (Fig 3C).

\section{Data of formulations unlisted in Japanese Orange Book}

In the dissolution profile of untreated hydroxyzine pamoate capsule (Fig 4A), less than $40 \%$ of the hydroxyzine dissolved in $10 \mathrm{~min}$ and most of them dissolved in $60 \mathrm{~min}$. Therefore this drug be-
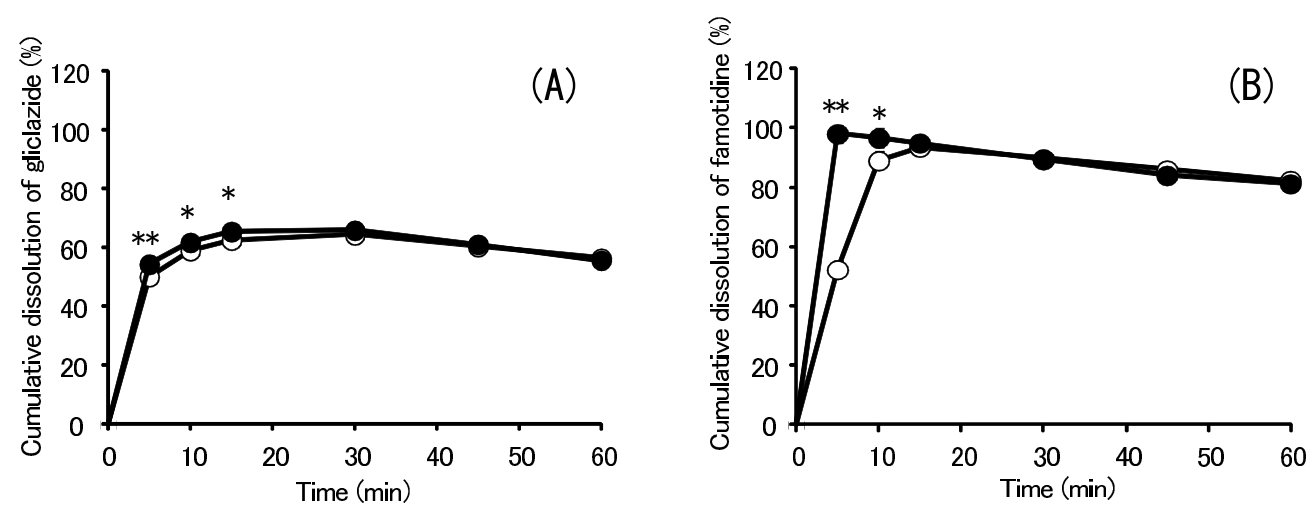

Fig 1 Dissolution profiles of gliclazide in Glimicron ${ }^{\circledR}$ - HA tablet $20 \mathrm{mg}(\mathrm{A})$ and famotidine in Gaster ${ }^{\circledR}$ tablet $10 \mathrm{mg}$ (B) Data are shown as the mean \pm SD $(n=4)$. Most error bars are not shown when the SD values fall within the symbols. Closed circles $(O)$ and open circle $(\bigcirc)$ means treated and untreated with the SSM, respectively. $* P<0.05, * * P<0.01$ : Significantly different compared to SSM untreated. Student's $t$-test. 

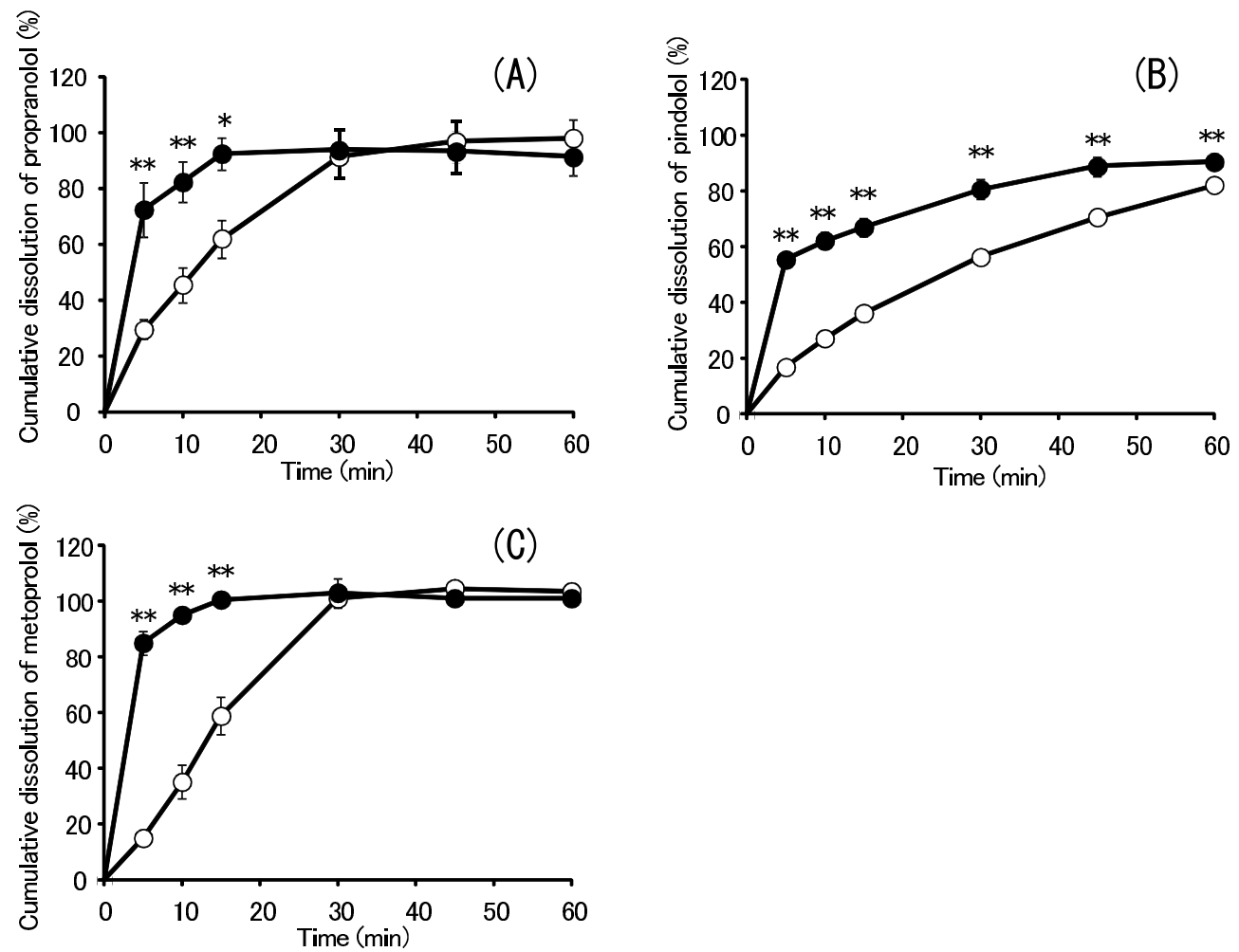

Fig 2 Dissolution profiles of propranolol in Inderal ${ }^{\circledR}$ tablet $10 \mathrm{mg}(\mathrm{A})$, pindolol in Carvisken ${ }^{\circledR}$ tablet $5 \mathrm{mg}$ (B) and metoprolol in Seloken ${ }^{\circledR}$ tablet $20 \mathrm{mg}(\mathrm{C})$

Data are shown as the mean \pm SD $(n=4)$. Some error bars are not shown when the SD values fall within the symbols. Closed circles $(O)$ and open circle $(\bigcirc)$ means treated and untreated with the SSM, respectively. $* P<0.05, * * P<0.01$ : Significantly different compared to SSM untreated. Student's $t$-test.

longs to the moderate-dissolution group. Treated hydroxyzine pamoate capsule dissolved rapidly same as other moderate-dissolution drugs. According to the Japanese Orange Book, hydroxyzine pamoate tablet $(25 \mathrm{mg}$ ) hardly dissolves in $15 \mathrm{~min}$, while gradually dissolving approximately $44 \%$ of the tablet in $60 \mathrm{~min}$ (Fig $\mathbf{4 A}$, dashed line). Given the same conditions, the dissolution behavior of hydroxyzine pamoate capsule $(25 \mathrm{mg})$ was found to significantly differ from that of hydroxyzine pamoate tablet: The capsule dissolved faster than the tablet.

In the dissolution profile of untreated phenytoin tablet (Fig 4B), less than 15\% of phenytoin dissolved in 10 min, and it was almost saturated in the solution at $30 \mathrm{~min}$. This result suggests that the phenytoin tablet belongs to the slow-dissolution group. Treated phenytoin tablet was rapidly dissolved within $5 \mathrm{~min}$, but the difference of solubility between treated and untreated with the SSM was reduced same as the dissolution profile of glimepiride (Fig 3C). On the other hand, a combined tablet of phenytoin $(67 \mathrm{mg})$ and phenobarbital $(33 \mathrm{mg}$ ) dissolved faster than a single tablet of phenytoin $(100 \mathrm{mg})$ : the combination tablet dissolved more than $80 \%$ at $60 \mathrm{~min}$ (Fig 4B, dashed line), the single tablet dissolved only $25 \%$ (Fig 4B, solid line).

\section{Discussion}

In previous studies, we examined the solubility of poorly water-soluble drugs that were treated with the SSM, and we reported that phenytoin and pranlukast ${ }^{10)}$ dissolved in the suspension to the saturated solubility. This suggests that the SSM 

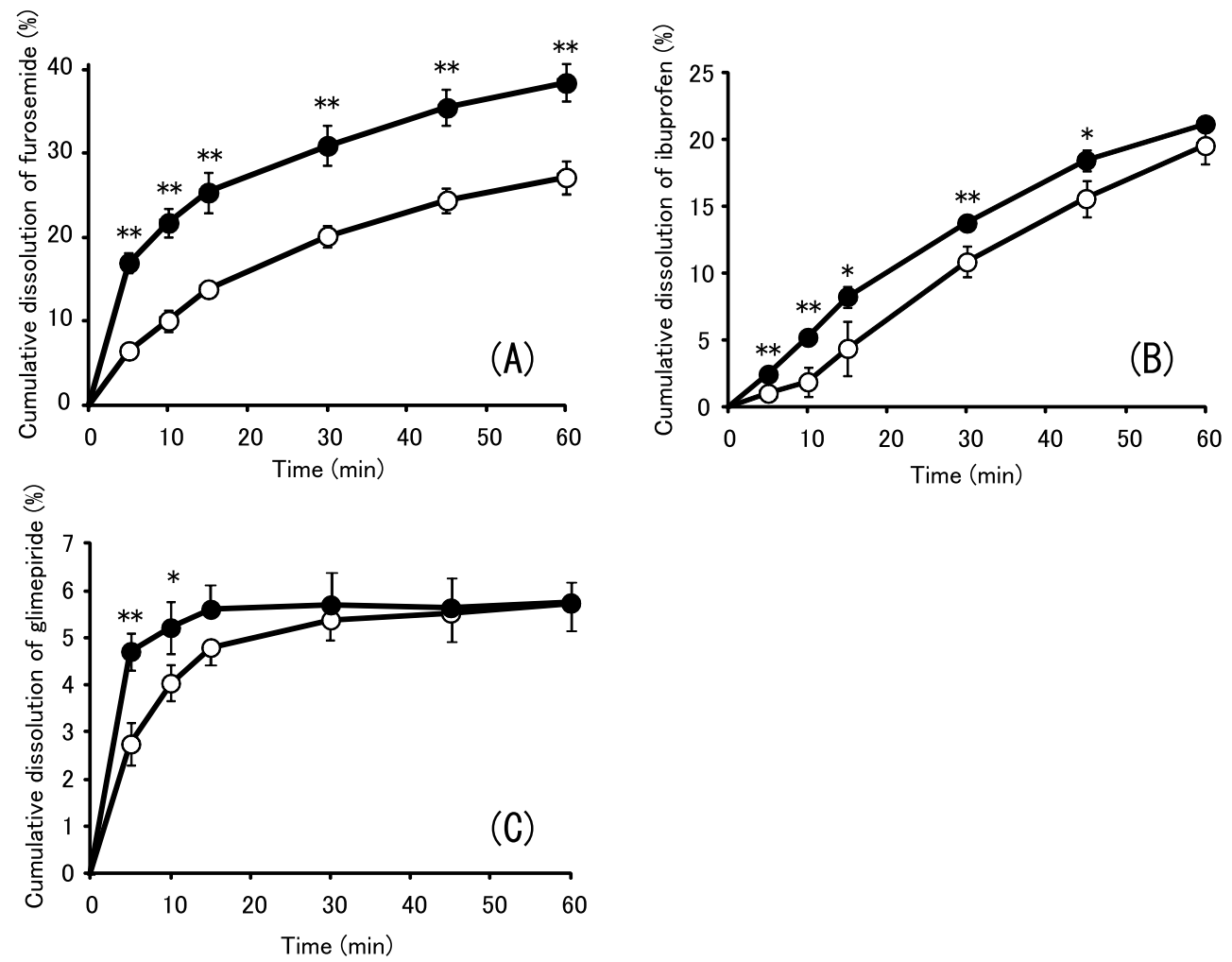

Fig 3 Dissolution profiles of furosemide in Lasix ${ }^{\circledR}$ tablet $20 \mathrm{mg}$ (A), ibuprofen in Brufen ${ }^{\circledR}$ tablet $100 \mathrm{mg}$ (B) and glimepiride in Amaryl ${ }^{\circledR}$ tablet $1 \mathrm{mg}(\mathrm{C})$

Data are shown as the mean \pm SD $(n=4)$. Some error bars are not shown when the SD values fall within the symbols. Closed circles $(\mathbf{O})$ and open circle $(\bigcirc)$ means treated and untreated with the SSM, respectively. $* P<0.05, * * P<0.01$ : Significantly different compared to SSM untreated. Student's $t$-test.
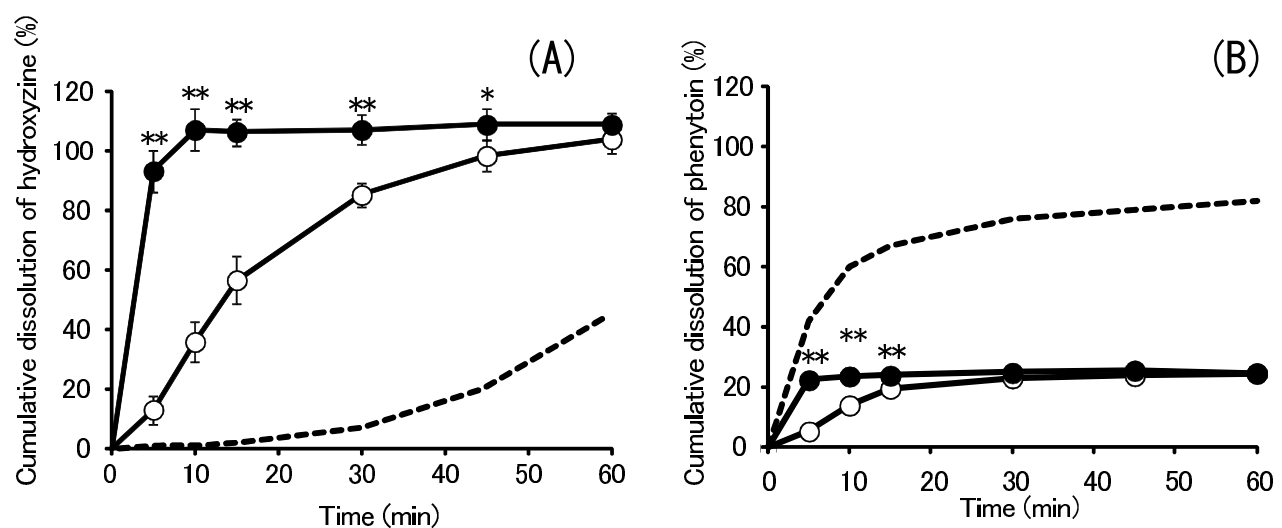

Fig 4 Dissolution profiles of hydroxyzine in Atarax ${ }^{\circledR}$-P capsule $25 \mathrm{mg}$ (A) and phenytoin in Aleviatin ${ }^{\circledR}$ tablet $100 \mathrm{mg}$ (B) Data are shown as the mean \pm SD $(n=4)$. Some error bars are not shown when the SD values fall within the symbols. Closed circles $(\mathbf{O})$ and open circle $(\bigcirc)$ means treated and untreated with the SSM, respectively. Dashed lines indicate the dissolution profiles of hydroxyzine pamoate tablet $(25 \mathrm{mg})$ (A) and phenytoin $(67 \mathrm{mg}) /$ phenobarbital $(33 \mathrm{mg}$ ) combination tablet (B) in 1st fluid, covered in the Japanese Orange Book. $* P<0.05, * * P<0.01$ : Significantly different compared to SSM untreated. Student's $t$-test.

may help increase the initial drug concentrations in the stomach, which seems unlikely by oral tablet/capsule administration, and may affect the absorbability of those drugs by the body. Nevertheless, the dissolution behavior of treated drugs with the SSM has not been confirmed. In this study, we compared the dissolution behavior of 10 drugs, treated vs untreated.

To determine whether to use the SSM to administer drugs to patients, pharmacists working 
for hospitals usually refer to guidebooks ${ }^{1)}$ on the disintegrity of suspended drugs, though they rarely search for the dissolution behavior of those drugs. This study found that the SSM significantly enhanced the dissolution rate for most of the tested drugs in $5 \mathrm{~min}$, with various degrees of enhancement. Notably, the SSM treatment significantly increased the dissolution rate of the beta-blockers, propranolol and metoprolol. Their elevated dissolution rates may increase the drug absorption rate, thereby excessively lowering the blood pressure.

It is impractical to perform dissolution tests for each drug. Thus, we used existing dissolution profiles found in interview forms and the Japanese Orange Book to consider the estimability of the effect of the SSM on the dissolution of each drug.

Figure 1 indicates that drugs in the rapid-dissolution group show similar dissolution behavior between treated and untreated with the SSM. The SSM promotes the disintegration and subsequent dissolution of the drugs, and it was clarified from the drug dissolution that the disintegration and dissolution of untreated drugs were also promoted in the solution as rapidly as for the treated drugs. The cumulative solubility of gliclazide and famotidine was found to become lower over time. This is probably because gliclazide and famotidine were decomposed in the 1st fluid with a $\mathrm{pH}$ of 1.2: They are known to be highly likely to be hydrolyzed in a strongly acid solution (http:// www2.jp-orangebook.gr.jp/data/06/06_02/06_ 02_Gliclazide.pdf, April 24, 2015, http://www2. jp-orangebook.gr.jp/data/06/06_06/06_06_Famotidine.pdf, April 24, 2015).

Figure 2 shows that drugs in the moderate-dissolution group were greatly affected by the SSM in the initial stage $(0-5 \mathrm{~min})$ of disintegration and dissolution; thus, the solubility of treated and untreated drugs was found to significantly differ between 5 and $15 \mathrm{~min}$. Furthermore, a significant difference was recognized between treated and untreated pindolol until 45 min (Fig 2B). The difference of dissolution behavior between treated and untreated drugs was greater for pindolol than for propranolol and metoprolol, probably because of the dissolution profile of the untreated drug: Only $67 \%$ of untreated pindolol dissolved in 30 min (Table 2).

Figure 3 shows that drugs in the slow-dissolution group were recognized as being little affected by the SSM in terms of solubility. The solubility of treated furosemide and ibuprofen was approximately 10 and 3 percentage points greater than untreated each drug in $10 \mathrm{~min}$, and the difference remained the same subsequently. The solubility of treated glimepiride was significantly greater than untreated glimepiride at 5 and $10 \mathrm{~min}$, however the difference disappeared after $15 \mathrm{~min}$. Such a difference profile of these drugs was recognized because furosemide and ibuprofen did not dissolve completely in $60 \mathrm{~min}$, whereas glimepiride, which is very poorly soluble, was almost saturated in the solution at $30 \mathrm{~min}$.

It should be noted that these findings are only comparisons of data on dissolution profiles; hence, it is too early to discuss the absorbability and adverse reactions of those drugs. There are few reports on variations in drug concentration in the blood for drugs administered by the SSM. Miyazaki et $a l^{11)}$ measured the tegafur concentrations in the blood of a patient who was treated with tegafur, gimeracil and oteracil potassium (TS- $1^{\circledast}$ combination capsule), and reported that the drug absorbability and maximum blood concentrations were higher for the SSM treatment form than for orally administered capsules (Hirata et $\left.a l^{12)}\right)$. The dissolution profiles of TS-1 ${ }^{\circledR}$ 
capsules, not listed on the Japanese Orange Book website, are listed on the interview forms of TS- $1^{\oplus}$ s generic drugs, including that of Esueewan ${ }^{\circledR}$ capsules. According to the interview forms, the solubilities of tegafur from a TS- $1^{\circledast}$ capsule $(25$ $\mathrm{mg}$ ) and an Esueewan ${ }^{\circledR}$ capsule $(25 \mathrm{mg})$ in a solution ( $\mathrm{pH} 1.2,50 \mathrm{rpm}$ ) are both approximately $80 \%$ in $5 \mathrm{~min}$ and $90 \%$ in $10 \mathrm{~min}$. Most of the formulations dissolved in $15 \mathrm{~min}$. Similar progress was recognized in gimeracil and oteracil potassium. From these results, it is estimated that the TS $-1^{\circledast}$ capsule falls in the rapid-dissolution group. The solubility of tegafur in water is regarded as $1.68 \mathrm{~g} / 100 \mathrm{~mL}$ at $20^{\circ} \mathrm{C}$ (Interview Form of TS- $1^{\oplus}$ combination capsule, revised $21 \mathrm{st}$ ed, ed by Taiho Pharmaceutical Co, Ltd, Tokyo, July 2014, http://www.info.pmda.go.jp/go/intervi ew/1/400107_4229101D1025_1_10E_1F, April $24,2015)$; therefore, tegafur in TS- $1^{\circledast}$ capsule form is considered to almost completely dissolve via the SSM, similarly to gliclazide (Fig 1A).

Using the SSM to suspend drugs omits the process of disintegration and initial dissolution of the formulation within the stomach. In this light, absorbability by digestive canal is seemingly affected more by tube-administered suspended drugs than by orally-administered solid formulations. In this study, we examined only several kinds of drugs about the rapid-, moderate- and slow-dissolution group, so it is necessary to examine the dissolution profile of more drugs. However, when suspended drugs that are categorized as the rapidor the moderate-dissolution groups are administered, the patients' body conditions should be carefully monitored. Adverse reactions (eg, hypotension after the administration of beta-blocker drugs) might occur by blood drug concentration increase.

As of January 10, 2015 the dissolution profiles for 688 pharmaceutical ingredients are provided in the Japanese Orange Book. Although the book covers a few thousand formulations/products, including different dosages and forms (eg, tablet, granule, powder) of the same ingredient, some newly developed drugs and some available drugs have yet to be listed. For example, hydroxyzine pamoate, commonly used clinically, is covered in the forms of tablet and dry syrup, but not capsule. According to the book, tablets of hydroxyzine pamoate tablets hardly dissolved within $30 \mathrm{~min}$, and do not start to gently dissolve until afterwards. From this, hydroxyzine tablets belong to the slow-dissolution group. In contrast, $40 \%$ of hydroxyzine pamoate capsule dissolved in $10 \mathrm{~min}$ and 100\% in $60 \mathrm{~min}$ (Fig 4A), from which hydroxyzine capsule belongs to the moderate-dissolution group. The SSM enhanced the dissolution of hydroxyzine, whose solubility was double that of untreated hydroxyzine at $15 \mathrm{~min}$. Such solubility increase continued to $45 \mathrm{~min}$.

An antiepileptic drug, phenytoin is listed only as a combination of phenytoin and phenobarbital, or as a combination of phenytoin, phenobarbital and caffeine-sodium benzoate. This study examined single-tablet phenytoin and compared its solubility with a phenobarbital-combined drug. Phenobarbital-combined phenytoin tablet was found to be more soluble, and to dissolve more rapidly, than phenytoin tablet (Fig $\mathbf{4 B}$ ). It is thought that $0.3 \%$ polysorbate 80 added as a solubilizer to the test solution stimulates dissolution (http://www2.jporangebook.gr.jp/data/07/07_06/07_06_Phenytoin_ Phenobarbital.pdf, April 24, 2015).

From those findings, it must be noted that the dissolution behavior may not conform to the behavior in the 1st fluid for dissolution test, particularly in cases where formulations ( $e g$, tablet, capsule, combination) unlisted in the book are 
administered via the SSM or where a solution contains some solubilizer.

This study pointed out for the first time that the dissolution behaviors of 10 drugs treated with the SSM can be estimated from the dissolution profiles listed in the Japanese Orange Book. The number of drug we examined is not enough, and it may still be difficult to estimate the blood drug concentrations of patients administered using the SSM, as well as the efficacy and safety. Nevertheless, it is safe to suggest that, when analyzing the possibility of the SSM treatment, pharmacists should be aware of drug dissolution profiles, consider every possible effect of regular drug administration on blood drug concentrations and pharmacological action, and regularly monitor the patients' conditions for adverse reactions.

\section{Acknowledgement}

The authors are grateful to $\mathrm{Mr}$ Akihito Momose, Mr Kensuke Mitsuhashi, Mr Hirotoki Sato, Mr Ryota Kanno and Mr Takuya Ashizaki for excellent technical assistance.

\section{Conflict of Interest}

The authors indicated no conflicts of interest.

\section{References}

1) Kurata N, "Simple suspension method - handbook for tube administration of oral medicines", ed by Fujishima I, Jiho Inc, Tokyo, 2006, pp1-47.

2) Nishizono K, Agatsuma H, Sasaki Y, Inase M, Kase Y, Andou T, Kurata N, The results of questionnaire about simple suspension method in Japanese Red Cross hospitals comparison between the January of 2006 year and the June of 2007 year, J Jpn Soc
Parenteral Enteral Nutr, 2009, 24, 595-598.

3) Shito T, Yamaura M, The introduction of simple suspension method to increase pharmacy practice efficiency, Jpn J Pharm Health Care Sci, 2006, 32, 517-522.

4) Jingami S, Miyazaki M, Matsumoto Y, Sadanaga A, Fukunaga E, Development of a dispensing support system for administration of suspensions of tablets and capsules by feeding tube, Jpn J Pharm Health Care Sci, 2008, 34, 333-340.

5) Yamamoto $Y$, Inoue $H$, Horibe $C$, Michima $N$, The role of the pharmacist in determining the method of administration of medicines in suspension though a feeding tube, Jpn J Drug Inform, 2011, 12, 117-122.

6) Yano K, Ikarashi N, Ito K, Orii T, Kurata N, Iida J, Sugiyama K, Comparison of the pravastatin original and generic drugs in the simple suspension method, Jpn J Pharm Health Care Sci, 2008, 34, 699-704.

7) Miyamoto E, Kawaguchi A, Hamaguchi N, Oshima T, Maida C, Saito K, Wakiya Y, Mutoh K, Kanamori $\mathrm{K}$, Evaluation of generic oral products using dissolution tests and the simple suspension method, Jpn J Pharm Health Care Sci, 2007, 33, 942-947.

8) Yano K, Takezawa T, Mochizuki T, Ikarashi N, Ito K, Orii T, Kurata N, Sugiyama K, Stability of drugs when prepared for tube administration by simple suspension method, Jpn J Pharm Health Care Sci, 2006, 32, 1094-1099.

9) Suryani N, Sugiyama E, Kurata N, Sato H, Stability of ester prodrugs with magnesium oxide using the simple suspension method, Jpn J Pharm Health Care Sci, 2013, 39, 375-380.

10) Kobayashi M, Takakura M, Noda K, Sakurada W, Tadano K, Comparison of solubility for poorly water-soluble proprietary and generic drugs in the simple suspension method, Jpn J Pharmaceu Sci Tech, 2014, 74, 93-98.

11) Miyazaki $Y$, Imamura $H$, Furukawa $H$, Tatsuta $M$, Yasui Y, Anami S, Yasui H, Pharmacokinetics of TS-1 Administered Through Jejunostomy for Unresectable advanced Remnant Gastric Cancer, Jpn J Gastroenterol Surg, 2010, 43, 214-219.

12) Hirata K, Horikoshi N, Aiba K, Okazaki M, Denno R, Sasaki K, Nakano Y, Ishizuka H, Yamada Y, Uno S, Taguchi T, Shirasaka T, Pharmacokinetic study of S-1 : a novel oral fluorouracil antitumor drug, Clin Cancer Res, 1999, 5, 2000-2005. 